\title{
Integrated positron emission tomography/computed tomography for evaluation of mediastinal lymph node staging of non-small-cell lung cancer in a tuberculosis- endemic area: A 5-year prospective observational study
}

\author{
J A Shaw, ${ }^{1} \mathrm{MB} \mathrm{ChB}$; E M Irusen, ${ }^{1} \mathrm{MB} \mathrm{ChB}, \mathrm{FCP}(\mathrm{SA}), \mathrm{PhD} ;$ F von Groote-Bidlingmaier, ${ }^{1} \mathrm{MD}$; \\ J M Warwick, ${ }^{2} \mathrm{BSc}, \mathrm{MB} \mathrm{ChB}, \mathrm{FCNP}(\mathrm{SA}), \mathrm{MMed}, \mathrm{PhD} ; \mathbf{B}$ Jeremic, ${ }^{3} \mathrm{MD}, \mathrm{PhD} ; \mathbf{R}$ du Toit, ${ }^{1} \mathrm{MB}$ ChB; \\ C F N Koegelenberg, ${ }^{1} \mathrm{MB} \mathrm{ChB}, \mathrm{MMed}$ (Int), FCP (SA), FRCP (Lond), Cert Pulm (SA), PhD

\begin{abstract}
${ }^{1}$ Division of Pulmonology, Department of Medicine, Faculty of Medicine and Health Sciences, Stellenbosch University and Tygerberg Academic Hospital, Tygerberg, Cape Town, South Africa

${ }^{2}$ Division of Nuclear Medicine, Department of Medical Imaging and Clinical Oncology, Faculty of Medicine and Health Sciences, Stellenbosch University and Tygerberg Academic Hospital, Tygerberg, Cape Town, South Africa

${ }^{3}$ Division of Radiation Oncology, Department of Medical Imaging and Clinical Oncology, Faculty of Medicine and Health Sciences, Stellenbosch University and Tygerberg Academic Hospital, Tygerberg, Cape Town, South Africa
\end{abstract}

Corresponding author: J A Shaw (jane.shaw@stonedragon.co.za)

Background. Integrated positron emission tomography/computed tomography (PET-CT) is a well-validated modality for assessing mediastinal lymph node metastasis in non-small-cell lung cancer (NSCLC), which determines management and predicts survival. Tuberculosis (TB) is known to lead to false-positive PET-CT findings.

Objectives. To assess the diagnostic accuracy of PET-CT in identifying mediastinal lymph node involvement of NSCLC in a high TB-endemic area.

Methods. Patients who underwent both PET-CT and lymph node tissue sampling for the investigation of suspected NSCLC were prospectively included in this observational study. Results were analysed per patient and per lymph node stage. A post-hoc analysis was performed to test the validity of a maximum standardised uptake value (SUVmax) cut-off for lymph node positivity.

Results. PET-CT had a sensitivity of $92.6 \%$, specificity of $48.6 \%$, positive predictive value of $56.8 \%$ and negative predictive value (NPV) of $90.0 \%$ in the per-patient analysis. Diagnostic accuracy was $67.2 \%$. Similar values were obtained in the per-lymph node stage analysis. TB was responsible for $21.1 \%$ of false-positive results. A SUVmax cut-off of 4.5 yielded an improvement in diagnostic accuracy from $64.0 \%$ to $84.7 \%$ compared with a cut-off of 2.5 , but at the cost of decreasing the NPV from $90.6 \%$ to $83.5 \%$.

Conclusion. In a high TB-endemic area, PET-CT remains a valuable method for excluding mediastinal lymph node involvement in NSCLC. Patients with a negative PET-CT may proceed to definitive management without further invasive procedures. However, PET-CT-positive lymph nodes require pathological confirmation, and the possibility of TB must be considered.

S Afr Med J 2015;105(2):145-150. DOI:10.7196/SAMJ.8357

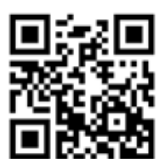

Lung cancer is the most common cause of death from cancer worldwide, with equally poor survival in developed and developing regions. ${ }^{[1]}$ Non-small-cell lung cancer (NSCLC) comprises $75-80 \%$ of lung cancer cases. ${ }^{[2]}$ Determining mediastinal lymph node involvement is fundamental in the staging of NSCLC and has been shown to have prognostic significance..$^{[3]}$ The node $(\mathrm{N})$ stage determines the treatment strategy, as patients with $\mathrm{N} 2$ lymph node metastasis are considered inoperable and only selected patients with this stage are eligible for chemotherapy and/or radiotherapy with curative intent. ${ }^{[4,5]}$

At present, integrated positron emission tomography/computed tomography (PET-CT) is recognised as being superior to PET alone, $\mathrm{CT}$ alone, or visual comparison of PET and CT images for the assessment of mediastinal lymph node involvement in NSCLC, ${ }^{[6]}$ with an average sensitivity, specificity, positive predictive value (PPV) and negative predictive value (NPV) of $73 \%, 80 \%, 78 \%$ and $91 \%$, respectively. ${ }^{[2]}$ This imaging modality has been available in the Western world since the 1990s, forming an integral part of mediastinal lymph node staging in those regions, ${ }^{[7]}$ but has only recently become standard practice in South Africa (SA). It has been suggested that negative PET-CT for mediastinal lymph node involvement requires no further invasive staging and surgical resection may proceed. ${ }^{[8]}$

Developing regions, particularly high tuberculosis (TB)-endemic areas, face the potential challenge of interpreting PET-CT images as false positive in TB-involved lymph nodes. ${ }^{[2,5,9,10]}$ The effect of TB on PET-CT staging of NSCLC has previously been investigated in areas with an incidence of TB of approximately 73/100 000 population. ${ }^{[5,11,12]}$ Two studies from Korea showed that the sensitivity (75\% and $46 \%$, respectively) and PPV (27\% and $72 \%)$ of PET-CT were adversely affected, but that a high specificity ( $85 \%$ and $98 \%)$ and NPV ( $98 \%$ in both studies) were maintained, especially when calcification and attenuation of nodes on CT was taken into account. ${ }^{[5,11]}$ Recent data from China demonstrated a similar pattern of results, with a relatively low sensitivity ( $61.8 \%$ and $78.6 \%$ for adenocarcinoma and squamous cell carcinoma, respectively) and specificity (66.3\% and $45.5 \%)$, and a high NPV ( $80.9 \%$ and $87 \%)$. The authors stated that the high incidence of granulomatous disease in their region impacted significantly on the rate of false-positive results in their study. ${ }^{[12]}$ According to the World Health Organization, the incidence of TB in SA is $1000 / 100000$ population. ${ }^{[13]}$ To date, no study of the efficacy 
of this modality for mediastinal lymph node staging in NSCLC in an area with such a high incidence of TB has been conducted.

The aim of this study was to assess the diagnostic accuracy of integrated PET-CT in detecting NSCLC metastases in mediastinal lymph nodes in patients from a high TB-endemic area.

\section{Methods}

Study design and patient population Tygerberg Academic Hospital (TAH), Cape Town, provides tertiary services for approximately 2 million people in the Western Cape Province of SA, draining the eastern subdistrict of the Cape Town metropole and surrounding areas. All patients referred with a suspicion of NSCLC to the Division of Pulmonology at TAH from January 2009 to December 2013 were eligible for inclusion in this observational study. Ethical approval for the study was granted by the Health Research
Ethics Committee of Stellenbosch University. Patients with an intermediate to high pretest probability of malignancy according to clinical guidelines ${ }^{[14]}$ were referred for PET-CT according to the standardised diagnostic algorithm that was used at the time of the study, with the PET-CT generally performed before tissue sampling (Fig. 1). Patients who had no lymph node tissue sampling performed were excluded. Patients were followed up to the point of referral for definitive treatment or palliation.

\section{PET-CT scanning}

All studies were performed from the base of skull to the upper thigh with a Siemens Biograph or Philips Gemini PETCT system with 16-slice uncontrasted CT (Fig. 2). The procedure was performed according to European Association of Nuclear Medicine guidelines. ${ }^{[15]}$ Patients fasted for at least 6 hours prior to the study.
Blood glucose levels were tested prior to the scan, which was postponed if the patient had a level of $>11.1 \mathrm{mmol} / \mathrm{L}$. Patients lay supine for 30 minutes prior to the study, and for a further 60 minutes, without talking, after injection of ${ }^{18}$ fluorodeoxyglucose (FDG). An FDG dose of $7-13 \mathrm{mCi}$ was administered according to body weight. All images were interpreted by an experienced nuclear medicine physician and at least one radiologist, and independently by a respiratory physician. Lymph node positivity was defined as any increase in FDG uptake compared with the mediastinal blood pool. Nodes were considered negative if they demonstrated a lower or equal FDG uptake compared with the mediastinal blood pool. The intensity of the FDG uptake in lymph nodes of interest was quantified using a Philips EBW workstation. A cursor was manually placed at the site of highest visual uptake within the lymph node, and

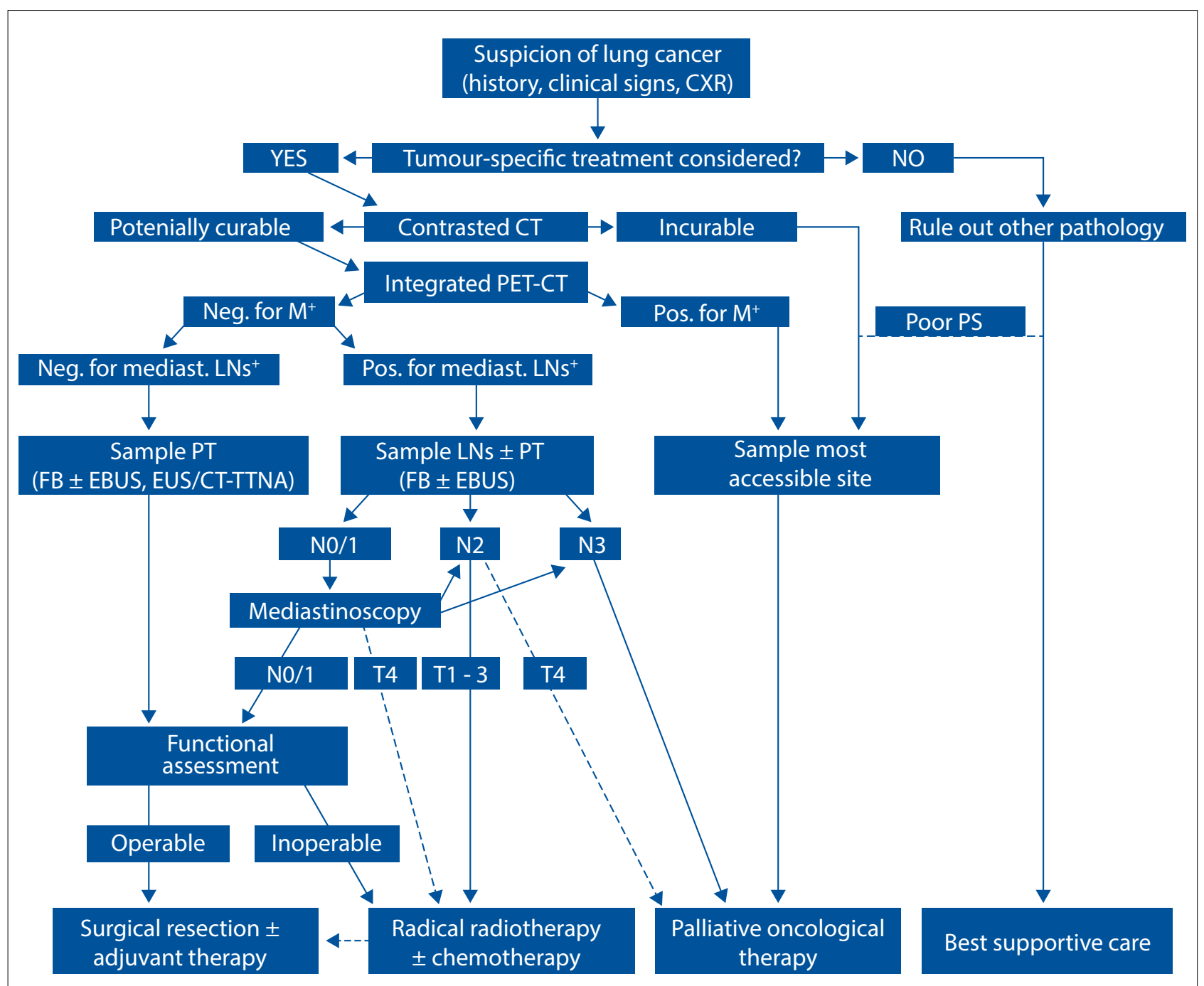

Fig. 1. Algorithm utilised during the study period for the evaluation of patients with suspected lung cancer. (CXR = chest radiograph; neg. = negative; pos. = positive; $M=$ distant metastases; mediast. $L N s=$ mediastinal lymph nodes; $P T=$ primary tumour; $F B=$ flexible bronchoscopy; $E B U S=$ endobronchial ultrasound; EUS = endoscopic ultrasound; TTNA = transthoracic needle aspiration; $N=$ lymph node metastases; $T$ = tumour stage.) 


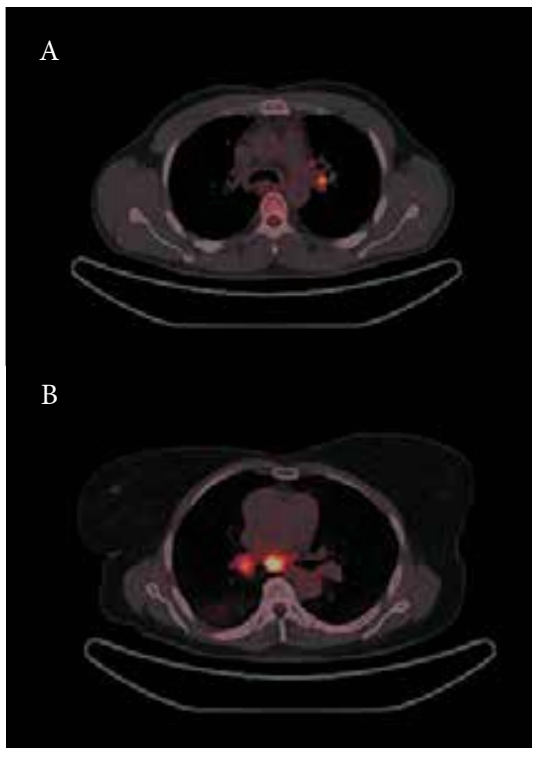

Fig. 2. (a) PET-CT scan demonstrating a left hilar lymph node with positivity due to proven tuberculosis. (b) PET-CT scan demonstrating a subcarinal lymph node with positivity due to metastatic non-small-cell lung cancer. the maximum standardised uptake value (SUVmax) was obtained from this slice using a circular region of interest with an $8 \mathrm{~mm}$ diameter. Lymph nodes were identified by accepted nomenclature according to the International Association for the Study of Lung Cancer lymph node map. ${ }^{[4]}$ These were further grouped into N1, N2 and N3 zones for prognostic purposes. ${ }^{[4]}$

\section{Tissue sampling}

Cells were obtained from lymph nodes of interest by an experienced interventional pulmonologist via endoscopic transbronchial needle aspiration, or fine-needle aspiration where accessible. Cytologically positive samples were considered to be evidence for the presence of metastatic malignancy, but negative samples were not considered sufficient evidence for the absence of malignancy.

Where further sampling was required, biopsy of suspicious lymph nodes was performed by mediastinoscopy. Alternatively, the entire lesion with surrounding lymph nodes was resected in an attempt at cure. All histological specimens thus obtained were considered definitive for both lymph node positivity and negativity. Biopsy specimens were concurrently tested for TB via staining for acid-fast bacilli.

Bronchial lavage and/or sputum samples were obtained from each patient and tested for TB via Ziehl-Neelsen staining (this being replaced by GeneXpert in 2013) and mycobacterial culture. These samples were also sent for routine microscopy, culture and sensitivity.

\section{Statistical aspects}

Descriptive statistics were performed. Analyses were conducted of data collected per patient, as well as per lymph node stage sampled (N1, N2, N3). The sensitivity, specificity, PPV, NPV, likelihood ratios (LRs) and diagnostic accuracy of PET-CT were calculated using standard methods.

A post-hoc secondary analysis of the SUVmax of all individual lymph nodes sampled was undertaken with the aim

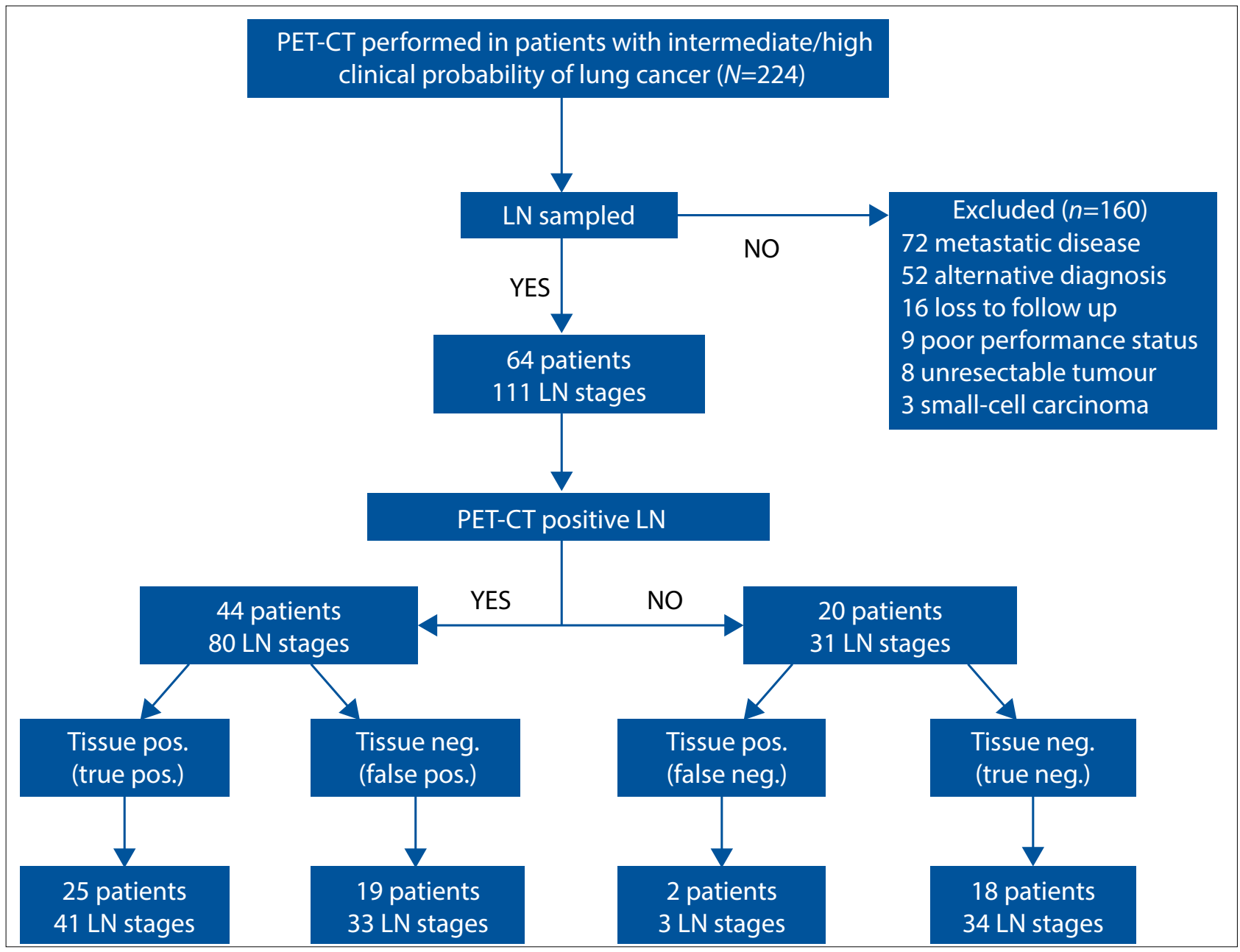

Fig. 3. Consort diagram. Alternative diagnoses included 21 patients with pulmonary infection of which 16 were tuberculosis, 28 patients with post-infectious changes with no organism identified, and 3 patients with non-carcinomatous tumours. $($ LN =lymph node; neg. = negative; pos. = positive. $)$ 
of identifying the SUVmax cut-off for designation of lymph nodes as 'positive' or 'negative' that would prove most clinically relevant. A receiver operating characteristic (ROC) curve was used to identify the SUVmax cut-off with the highest diagnostic accuracy. The SUVmax cut-off of 2.5 has been used in previous studies as a comparison value. It was therefore subsequently compared with the value identified by the ROC curve with regard to sensitivity, specificity, PPV, NPV and diagnostic accuracy. ${ }^{[16]}$

\section{Results \\ Study population}

During the study period, a total of 224 patients were referred for PET-CT based on an intermediate or high pre-test probability of lung cancer (Fig. 3). A total of 64 patients underwent extensive lymph node sampling and were therefore included in the study, with 111 separate lymph node stages sampled. Demographic data are depicted in Table 1.

\section{PET-CT findings}

In the per-patient analysis, PET-CT was found to have a sensitivity of $92.6 \%$, specificity of $48.6 \%$, PPV of $56.8 \%$, NPV of $90.0 \%$, positive LR of 1.8 and negative LR of 0.15 (Table 2). The diagnostic accuracy was $67.2 \%$. Of the false positives, $21.1 \%$ were attributed to active TB infection and $78.9 \%$ were reactive lymph nodes. In the perlymph node stage analysis, the sensitivity was $93.2 \%$, specificity $50.0 \%$, PPV $55.4 \%$ and NPV 91.7\%, with a positive LR of 1.9 , and negative LR of 0.14. Diagnostic accuracy was $67.6 \%$.

In the secondary analysis of SUVmaxes of individual nodes, the ROC curve identified a SUVmax cut-off of 4.5 as offering the highest overall diagnostic accuracy (Fig. 4). The comparison SUVmax cut-off of 2.5 was analysed and found to have a sensitivity of $95.7 \%$, specificity of $36.3 \%$, PPV of $56.8 \%$ and NPV of $90.6 \%$ when applied to these data. The diagnostic accuracy was $64.0 \%$. A SUVmax cut-off of 4.5 yielded a sensitivity of $80.0 \%$, specificity of $88.8 \%$, PPV of $86.2 \%$ and NPV of $83.5 \%$ (Table 3). The diagnostic accuracy was $84.7 \%$.

\section{Discussion}

In this first study of PET-CT for evaluation of mediastinal lymph node staging in NSCLC in SA, we have demonstrated that PET-CT had a high sensitivity of $92.6 \%$ and $93.2 \%$ per patient and per lymph node stage, respectively, and a high NPV of $90 \%$ and $91.7 \%$. It had a low specificity of $48.6 \%$ and $50.0 \%$ and a low PPV of $56.8 \%$ and $55.4 \%$. Moreover, we found favourable negative LRs. The diagnostic accuracy was $67.2 \%$ and $67.6 \%$, respectively. More than $20 \%$ of false-positive PET-CT scan results were attributable to active $\mathrm{TB}$, once again highlighting that TB can mimic lung cancer in the clinical setting.

These results confirm the value of PETCT for excluding lymph node involvement in NSCLC in a high TB-endemic area. The role of PET-CT can therefore be considered the same as in non-TB-endemic areas, where it is used to direct node biopsy rather than to aid in diagnosis. A patient with a PET-CT that is negative for any lymph node involvement may be considered to be accurately staged and therefore proceed to definitive management of the cancer without further invasive staging investigations. Importantly, in the SA context a positive lymph node on PETCT has an approximately $50 \%$ chance of

Table 1. Demographic data: characteristics of the population screened during the study period, with patients included in the study compared with patients excluded

\begin{tabular}{lll}
\hline & Study population & Patients excluded \\
\hline $\begin{array}{l}\text { Males, } n \text { (\%) } \\
\text { Females, } n \text { (\%) }\end{array}$ & $39(60.9)$ & $96(60.0)$ \\
Age range (years), $n(\%)$ & $25(39.1)$ & $64(40.0)$ \\
$\quad<40$ & $2(3.1)$ & $5(3.1)$ \\
$40-50$ & $11(17.2)$ & $17(10.6)$ \\
51 - 60 & $21(32.8)$ & $52(32.5)$ \\
$61-70$ & $20(31.3)$ & $49(30.6)$ \\
71 - 80 & $10(15.6)$ & $32(20.0)$ \\
$>80$ & $0(0.0)$ & $5(3.1)$ \\
Mean age (years) (standard deviation) & $59.3(10.2)$ & $61.6(10.7)$ \\
Histological type*, $n$ (\%) & & \\
Adenocarcinoma & $34(59.7)$ & $49(45.4)$ \\
Squamous cell carcinoma & $16(28.1)$ & $38(35.2)$ \\
Undifferentiated large-cell carcinoma & $4(7.0)$ & $17(15.7)$ \\
Other & $3(5.3)$ & $1(0.9)$ \\
Clinical stage* $n$ (\%) & & \\
Stage I & $10(17.5)$ & $3(2.9)$ \\
Stage II & $18(31.6)$ & $4(3.8)$ \\
Stage III & $22(38.6)$ & $41(39.0)$ \\
Stage IV & $7(12.3)$ & $57(54.3)$ \\
*In patients with proven NSCLC. & & \\
& &
\end{tabular}

Table 2. PET-CT results: data acquired from the comparison of PET-CT findings and tissue sampling, grouped per patient and per lymph node stage, and statistical analysis thereof

\begin{tabular}{lll}
\hline & Per-patient analysis & Per-lymph node stage analysis \\
\hline True positive, $n(\%)$ & $25(39.1)$ & $41(36.9)$ \\
True negative, $n(\%)$ & $18(28.1)$ & $34(30.6)$ \\
False positive, $n(\%)$ & $19(29.7)$ & $33(29.7)$ \\
False negative, $n(\%)$ & $2(3.1)$ & $3(2.7)$ \\
Sensitivity (95\% CI) & $92.6(0.743-0.987)$ & $93.2(0.803-0.982)$ \\
Specificity (95\% CI) & $48.6(0.322-0.653)$ & $50.0(0.376-0.624)$ \\
PPV (95\% CI) & $56.8(0.411-0.713)$ & $55.4(0.434-0.668)$ \\
NPV (95\% CI) & $90.0(0.669-0.983)$ & $91.7(0.764-0.978)$ \\
Positive likelihood ratio $(95 \% \mathrm{CI})$ & $1.803(1.295-2.511)$ & $1.864(1.445-2.403)$ \\
Negative likelihood ratio $(95 \% \mathrm{CI})$ & $0.152(0.038-0.611)$ & $0.136(0.044-0.419)$ \\
CI = confidence interval. & &
\end{tabular}


being a false positive. All such patients therefore require further investigations for pathological confirmation before they can be accurately staged and appropriately managed. Initial studies involving this modality in other regions of the world found a pooled average sensitivity, specificity, PPV and NPV of $73 \%, 80 \%, 78 \%$ and $91 \%$, respectively. ${ }^{[2]}$ The present study in

a high TB-endemic region demonstrated the anticipated reduction in specificity and PPV, which is attributable to the high rate of false-positive TB-infected or reactive nodes. However, our sensitivity and NPV are equal or superior to these international values. We postulate that the higher rate of pick-up of true-negative nodes in our study may be due to our method of combined analysis of PET-

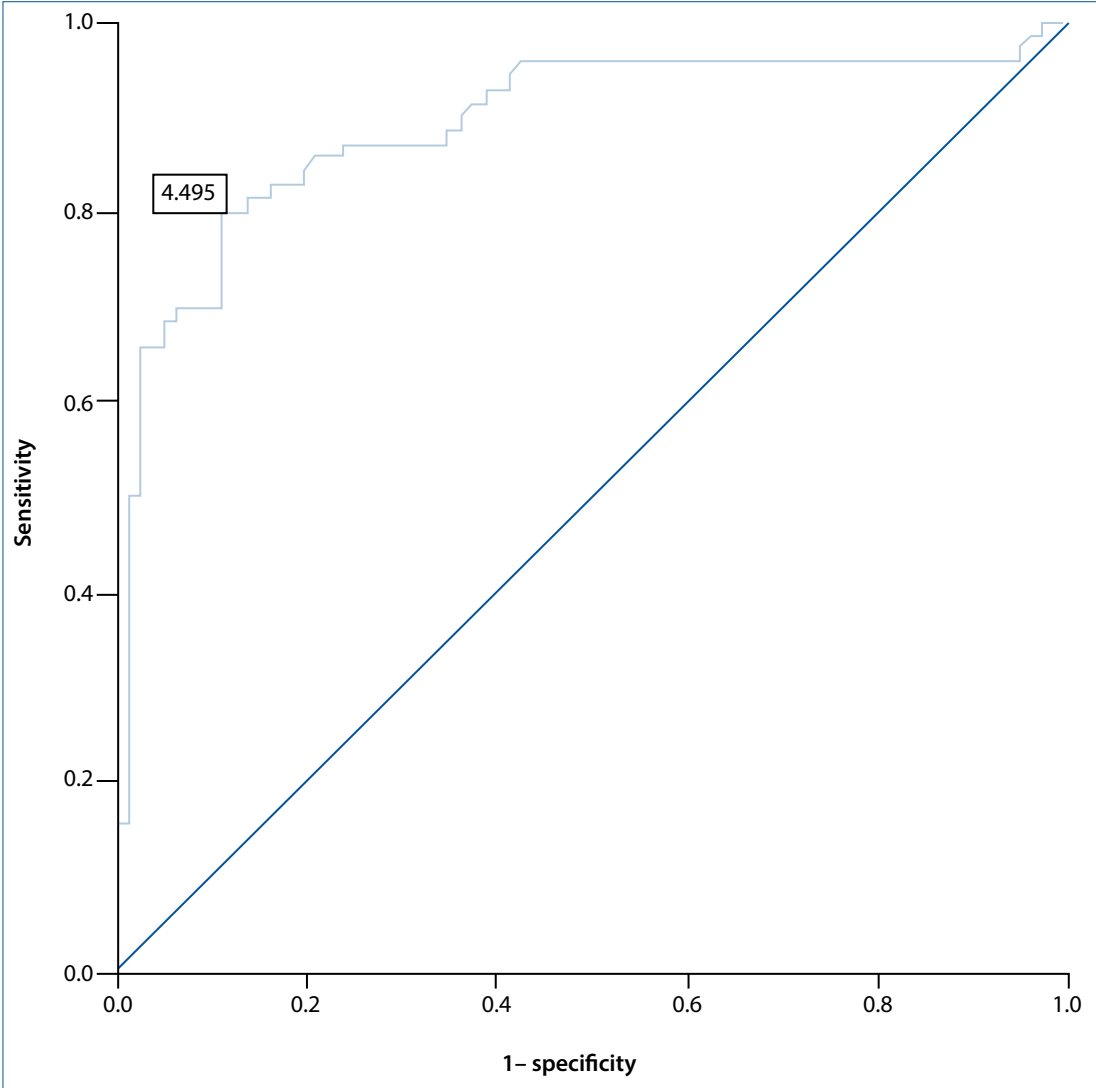

Fig. 4. ROC curve of SUVmaxes of individual lymph nodes. The value 4.495 is identified as having the highest overall diagnostic accuracy.

Table 3. Proposed SUVmax cut-off value results: data acquired from grouping individually sampled lymph nodes as positive or negative according to proposed SUVmax cut-off values of 2.5 and 4.5 (identified by ROC curve in the present study), and statistical analysis thereof

\begin{tabular}{lll}
\hline & SUVmax cut-off 2.5 & SUVmax cut-off 4.5 \\
\hline True positive, $n(\%)$ & $67(44.7)$ & $56(37.3)$ \\
True negative, $n(\%)$ & $29(19.3)$ & $71(47.3)$ \\
False positive, $n(\%)$ & $51(34.0)$ & $9(6.0)$ \\
False negative, $n(\%)$ & $3(2.0)$ & $14(9.3)$ \\
Sensitivity (95\% CI) & $95.7(0.872-0.989)$ & $80.0(0.684-0.883)$ \\
Specificity (95\% CI) & $36.3(0.260-0.478)$ & $88.8(0.792-0.944)$ \\
PPV (95\% CI) & $56.8(0.474-0.658)$ & $86.2(0.748-0.931)$ \\
NPV (95\% CI) & $90.6(0.738-0.976)$ & $83.5(0.736-0.904)$ \\
Positive likelihood ratio (95\% CI) & $1.501(1.264-1.784)$ & $7.111(3.801-13.306)$ \\
Negative likelihood ratio (95\% CI) & $0.118(0.037-0.373)$ & $0.225(0.141-0.361)$ \\
CI = confidence interval. & &
\end{tabular}

CT images by a nuclear medicine physician and a radiologist, who take into account their own judgement of the appearance of the lymph nodes and attenuation of the nodes on CT, as well as interpreting the PET. Studies have demonstrated that lymph nodes with higher attenuation ( $>70$ Hounsfield units) or calcification on CT have a much higher probability of being benign. Using these additional criteria for interpretation of images on PET-CT improves diagnostic accuracy, even in a TB-endemic area. ${ }^{[5,11,12]}$

Previous studies conducted in high TB-endemic areas did not demonstrate the anticipated reduction in specificity. In fact, these studies found a reduction in sensitivity. ${ }^{[5,11,12]}$ These authors proposed that this may have been due to the selective inclusion of patients of lower clinical stage with possible micrometastases to lymph nodes that did not demonstrate positivity on PET-CT, as well as the designation of all calcified lymph nodes as benign. It is worth considering that the present study was inadvertently subject to the same selective inclusion, although not to the same degree, and did not suffer corresponding losses in sensitivity. The present study does, however, agree with the previous studies $^{[5,11,12]}$ in the reduction of PPV, with preservation of NPV.

An ROC curve has been utilised in previous studies to identify the SUVmax cut-off with the highest diagnostic accuracy for the prediction of malignancy in mediastinal lymph nodes on PET-CT. Values identified in these studies included 4.4 and 5.3, yielding a diagnostic accuracy of $92 \%$ and $98 \%$, respectively. ${ }^{[16,17]}$ Although our post-hoc analysis of proposed SUVmax cut-offs suggested that a cut-off of 4.5 conferred an improvement in diagnostic accuracy from $64.0 \%$ to $84.7 \%$ compared with a cut-off of 2.5 , it came at the cost of decreasing the sensitivity from $95.7 \%$ to $80.0 \%$ and the NPV from $90.6 \%$ to $83.5 \%$. As PET-CT is used to exclude mediastinal spread rather than diagnose it, the reduction in NPV renders the cut-off of 4.5 less appropriate for clinical use.

The strength of the present study lies in the substantial number of lymph nodes sampled, and the prospective method in which the data were collected. A potential limitation was the necessary exclusion of a large number of patients - especially those in higher clinical stages of disease - prior to lymph node sampling. However, this was unavoidable as the study was conducted within the parameters of a normal clinical setting, and all decisions regarding invasive investigations were made in the interests of providing best patient care. 


\section{Conclusion}

PET-CT remains a valuable method for excluding mediastinal lymph node metastases in the staging of NSCLC in a high TB-endemic area, because of its high sensitivity and NPV. A patient with negative PETCT for mediastinal lymph node involvement may therefore safely and swiftly proceed to curative surgical intervention without undergoing further invasive staging procedures. However, the low specificity and PPV indicate that the finding of a positive lymph node on this imaging modality requires pathological confirmation, and that the possibility of TB must be considered.

\section{References}

1. Ferlay J, Soerjomataram I, Ervik M, et al. GLOBOCAN 2012 v1.0, Cancer Incidence and Mortality Worldwide: IARC CancerBase No. 11. Lyon: International Agency for Research on Cancer, 2013. http:// globocan.iarc.fr (accessed 9 February 2014)

2. De Wever W, Stroobants S, Coolen J, Verschakelen JA. Integrated PET/CT in the staging of non-smallcell lung cancer: Technical aspects and clinical integration. Eur Respir J 2009;33(1):201-212. [http:// cell lung cancer: Technical aspects and
dx.doi.org/10.1183/09031936.00035108]

3. Detterbeck FC, Postmus PE, Tanoue LT. The stage classification of lung cancer. Diagnosis and management of lung cancer, 3rd ed. American College of Chest Physicians evidence-based clinica practice guideline. Chest 2013;143(5, suppl):e191S-e210S [http://dx.doi.org/10.1378/chest.12-2354 4. Detterbeck FC, Boffa DJ, Tanoue LT. The new lung cancer staging system. Chest 2009;136(1):260-271 [http://dx.doi.org/10.1378/chest.08-0978]

5. Lee JW, Kim BS, Lee DS, et al. 18F-FDG PET/CT in mediastinal lymph node staging of non-smallcell lung cancer in a tuberculosis-endemic country: Consideration of lymph node calcification an distribution pattern to improve specificity. Eur J Nucl Med Mol Imaging 2009;36(11):1794-1802 [http://dx.doi.org/10.1007/s00259-009-1155-4]

6. Lardinois D, Weder W, Hany TF, et al. Staging of non-small-cell lung cancer with integrated positronemission tomography and computed tomography. N Engl J Med 2003:348(25):2500-2507 [http./ dx.doi.org/10.1056/nejmoa022136]
7. Beyer T, Townsend DW, Brun T, et al. A combined PET/CT scanner for clinical oncology. J Nucl Med 2000;41(8):1369-1379.

8. Perigaud C, Bridji B, Roussel JC, et al. Prospective preoperative mediastinal lymph node staging by integrated positron emission tomography-computerised tomography in patients with nonsmall-cell lung cancer. Eur J Cardiothorac Surg 2009;36(4):731-736. [http://dx.doi.org/10.1016/j. ejcts. 2009.05.044]

9. Chang JM, Lee HJ, Goo JM, et al. False positive and false negative FDG-PET scans in various thoracic diseases. Korean J Radiol 2006;7(1):57-69. [http://dx.doi.org/10.3348/kir.2006.7.1.57]

10. Sathekge MM, Maes A, Pottel H, Stolz A, van de Wiele C. Dual time-point FDG PET/CT for differentiating benign from malignant solitary pulmonary nodules in a TB-endemic area. S Afr Med J 2010;100(9):598-601

11. Kim YK, Lee KS, Kim BT, et al. Mediastinal nodal staging of non-small-cell lung cancer using integrated 18F-FDG PET/CT in a tuberculosis-endemic country: Diagnostic efficacy in 674 patients. Cancer 2007;109(6):1068-1077. [http://dx.doi.org/10.1002/cncr.22518]

12. Lu P, Sun Y, Sun Y, Yu L. The role of $18 \mathrm{~F}-$ FDG PET/CT for evaluation of metastatic mediastinal lymph nodes in patients with lung squamous-cell carcinoma or adenocarcinoma. Lung Cancer 2014;85(1):5358. [http://dx.doi.org/10.1016/j.lungcan.2014.04.004]

13. World Health Organization. Global Tuberculosis Report 2013. Geneva: World Health Organisation, 2013. http://www.who.int/tb/publications/global_report/en/(accessed 9 February 2014).

14. Gould MK, Donington J, Lynch WR, et al. Evaluation of individuals with pulmonary nodules: When is it lung cancer? Diagnosis and management of lung cancer, 3rd ed. American College of Chest Physicians evidence-based clinical practice guideline. Chest 2013;143(5, suppl):e78S-e92S. [http:// dx.doi.org/:10.1378/chest.12-2350]

15. Boellaard R, O'Doherty MJ, Weber WA, et al. FDG PET and PET/CT: EANM procedure guidelines for tumour PET imaging: version 1.0. Eur J Nucl Med Mol Imaging 2010;37(1):181-200. [http://dx.doi. org/10.1007/S00259-009-1297-4]

16. Bryant AS, Cerfolio RJ, Klemm KM, Buddhiwardhan O. Maximum standard uptake value of mediastinal lymph nodes on integrated FDG-PET-CT predicts pathology in patients with non-small cell lung cancer. Ann Thorac Surg 2006;82(2):417-423. [http://dx.doi.org/10.1016/j.athoracsur.2005.12.047]

17. Vansteenkiste JF, Stroobants SG, de Leyn PR, et al. Lymph node staging in non-small-cell lung cancer with FDG-PET scan: A prospective study on 690 lymph node stations from 68 patients. J Clin Oncol $1998 ; 16(6): 2142-2149$

Accepted 28 July 2014 